\title{
When Science is Not Enough: A Framework Towards More Customer-Focused Drug Development
}

\author{
Nektarios Oraiopoulos · William C. N. Dunlop
}

Received: April 20, 2017 / Published online: June 17, 2017

(C) The Author(s) 2017. This article is an open access publication

\begin{abstract}
Introduction: The purpose of this study was to identify the key barriers to a customer-focused drug development process and develop a comprehensive framework to overcome them.

Methods: The paper draws on existing literature, both academic and practitioner, across a range of disciplines (innovation management, marketing, organizational behavior, behavioral economics, health economics, industry reports). On the basis of this extensive review, a conceptual framework is developed that offers concrete suggestions on how organizations can overcome the barriers and enable a more customer-focused development process.

Results: The barriers to collaboration are organized into three distinct categories (economic, behavioral, organizational), and within each category, a one-to-one mapping between barriers and solutions is developed.
\end{abstract}

Enhanced content To view enhanced content for this article go to http://www.medengine.com/Redeem/ 8598F06012A083E4.

N. Oraiopoulos

Cambridge Judge Business School, University

of Cambridge, Cambridge, UK

W. C. N. Dunlop ( $₫)$

Mundipharma International, Cambridge, UK

e-mail: will.dunlop@mundipharma.com
Conclusion: The framework is specifically designed with the objective of offering actionable and practical advice to executives who face these challenges in their organizations. The paper provides a unique theoretical contribution by synthesizing findings from several academic disciplines with concrete examples from the pharmaceutical industry.

Funding: Mundipharma International Limited.

Keywords: Business; Customer-focused development; Drug development; Finance; Health economics; Market access; Patient access; Pharmaceuticals; R\&D management; Value frameworks

\section{INTRODUCTION}

On 18 October 2007, Pfizer announced the withdrawal of its inhaled insulin brand Exubera ${ }^{\circledR}$ from the market. Just a few months earlier, the company predicted the drug would be a US $\$ 2$ billion-a-year product. Yet, at the time the withdrawal decision was taken, Exubera had barely reached sales of US $\$ 12$ million, and it had cost Pfizer over US\$2.8 billion [1]. It is not uncommon for pharmaceutical companies to terminate drugs and write off substantial investments during clinical trials (and occasionally after market launch) owing to safety concerns. But what really made Exubera stand out as one of the most unprecedented and 
stunning failures in the history of the pharmaceutical industry [1] was the fact that the withdrawal decision was entirely based on disappointing sales, rather than any safety or efficacy concerns [2].

One unique feature of the pharmaceutical industry is that the patient who receives the drug is rarely the person who decides on that treatment or even pays for it. Instead, prescribing decisions in developed healthcare markets are typically made by physicians, and the payments are often made by insurance companies, government, or other organizations, with little or no payment directly from the patient. This creates a unique environment in which a successful commercialization strategy has to meet not only the regulatory requirements of the Food and Drug Administration (FDA) or European Medicines Agency (EMA) but also the requirements of the three end customers: the patient, physician, and payer. The term "payer" broadly refers to the healthcare budget holder and can include, e.g., Medicare in the USA. A high profile European organization, which reviews therapies from a cost perspective and provides guidance, is the UK National Institute for Health and Care Excellence (NICE). Traditionally, pharmaceutical companies have launched blockbuster drugs by just focusing on patients and physicians. This is no longer the case: given the ever rising reimbursement hurdle, a successful commercialization strategy relies on a sound understanding of the payer's needs and priorities. This aspect has been overlooked in the existing academic literature. In this article, we develop a framework to address this gap and enable organizations to better align their drug development process with the expectations of the end customer, and more specifically, the payer.

Consider again the case of Exubera, a drug that faced significant opposition from payers. In the USA, many insurance companies refused to cover treatment because Exubera was more expensive than existing treatments: US\$5 compared with US\$2-3 for injectable insulin [3]. In the UK, NICE argued that Exubera should only be approved for diabetics who had a proven fear of needles, as it offered no advantage over existing treatments [3]. The Exubera story is by no means unique. In recent years, there have been several drugs that met regulatory safety and efficacy hurdles, but failed to meet payer hurdles, e.g., Avastin ${ }^{\circledR}$ [4] and Zaltrap ${ }^{\circledR}$ [5].

Executives in pharmaceutical companies are well aware of the increasing role of the payer, and the fact that regulatory approval can no longer be considered a guarantee for market success and profitability. According to Harrison [6], 24\% of drug failures in phase II and phase III can be attributed to commercial and strategy reasons. Given that the average development cost for a new drug is estimated at US\$1.4 billion (out-of-pocket) and US\$2.55 billion (capitalized) dollars [7], anticipating and avoiding such market failures earlier in the process could potentially save companies hundreds of millions of dollars in research and development (R\&D) costs. However, to achieve this, close collaboration between the R\&D and commercial teams is critical. Such a collaboration requires that the project initiation and continuation decisions are made using the best possible information, rather than subjective and biased estimates. Numerous academic studies have shown how better communication between the commercial and R\&D teams can lead to projects with a higher success rate, a greater percentage of sales coming from new products, and a higher likelihood of successfully delivering the company's strategic goals (see [8] and the references therein). As a result, the extant academic literature highlights the importance of having a cross-functional team that brings together experts from various departments and ensuring that the project progresses to meet both the technical and commercial requirements $[9,10]$.

However, for most pharmaceutical companies, and despite the growing realization about the importance of the payer, the flow of information between the $R \& D$ and commercial teams is not always optimized. For example, development teams might not always include appropriate comparators (e.g., existing standards of care) in the clinical trials, but rather rely on placebos. This might be acceptable for regulatory approval by the FDA or EMA, but it is often not sufficient for the payer, who is likely 
to require evidence of direct comparison with the existing standards of care (when applicable). As a result, a number of projects that are unlikely to meet the customer's expectations continue to consume valuable resources as a result of biased information and organizational inefficiencies. By its very nature, developing new drugs will remain a highly risky and complex process. Expensive failures are an inherent part of medical discoveries, and that is why it is so critical for organizations to identify these failures as fast and efficiently as possible. This is precisely why maintaining a focus on the patients' and broader societies' true needs becomes even more important. This challenge is equally critical for drugs that are developed in-house (by the internal R\&D team of the organization) or are in-licensed from external organizations (e.g., through various types of partnerships).

The goal of this study was to develop a framework that would enable a better communication flow, and develop tangible suggestions on how to make the drug development process more customer-focused. The first step was to identify key barriers to cross-functional collaboration that have traditionally prevented an integrated development process in the pharmaceutical industry. To better understand the underlying causes of these barriers, they were categorized into those driven by economical, behavioral, or organizational reasons. For each category, an extensive literature review was conducted to identify the most effective management practices to overcome the specific challenges. This review covered a number of academic papers that spanned a wide spectrum of research (from organizational and behavioral economics to innovation management and organizational theory). For each literature-based solution, specific examples on how the proposed solution can be applied to the drug development process are provided. The central proposition of this work is that overcoming these barriers will allow pharmaceutical companies to prioritize projects that are more customer-focused and, therefore, generate more value (both for the companies and the patients). This article does not contain any new studies with human or animal subjects performed by any of the authors.

\section{CHALLENGES TO COLLABORATION AND COMMUNICATION}

\section{Economic}

The first and foremost characteristic of pharmaceutical R\&D is its highly uncertain nature, manifested directly in the high failure rates of new drugs. Developing new products is challenging and entails a great deal of risk for most industries [11]. Even so, the attrition rates of new pharmaceutical products are at a different order of magnitude compared even with the riskiest industries: for those drugs that successfully reach clinical testing (testing in humans) fewer than one in five (around 16\%) will receive regulatory approval [12]. These high attrition rates have been relatively stable across the past few decades, unlike the cost of conducting clinical trials, which has consistently increased [13].

The high attrition rates mean that pharmaceutical $R \& D$ teams are often susceptible to so-called progression-driven behavior where they focus their efforts on advancing a compound to the next stage without first ensuring that this is the right decision. However, in many cases, potential failures could be detected earlier. A recent study by Pfizer examined the decision-making processes behind 44 development programs [14]. A striking finding was that all programs that had successfully completed clinical proof of concept (PoC) could provide clear evidence that proper testing procedures had been followed in previous stages. On the contrary, for almost half (43\%) of those that failed at PoC, there was little evidence that all the necessary testing procedures had been followed. Had proper testing taken place, these failures would have been detected much earlier, saving the company millions of dollars. The need for superior clinical outcomes against the standard of care is even greater when a price premium is required to meet the cost of development. The existence of this progression-driven behavior is also highlighted in an extensive longitudinal study of AstraZeneca's small-molecule pipeline that examined the root causes behind project failures [15]. A possible explanation, suggested by the authors, is that 
"the use of volume-based metrics encouraged project teams and leadership groups to progress projects to the next phase in order to meet yearly goals".

A second characteristic of the drug development process that further complicates collaboration and efficient decision-making is its long development cycles. Even if we exclude the discovery phase of a new compound, it still takes 6-7 years from filing of a new molecular entity to getting regulatory approval (Pharmaceutical Research and Manufacturers of America [16]). During these lengthy cycles, a number of factors can separate the actions of an individual or group from the end goal of the project: market launch. In addition, given the highly specialized nature of the tasks and activities involved, this process is highly fragmented: different groups in the company, or even different companies (e.g., contract research organizations), are involved at different stages. As a result, scientists and managers are often rewarded on the basis of interim goals [17]. While such a reward scheme might seem understandable, given the lengthy development times, a by-product of these interim goals is that managers are often held accountable for reaching only a specific milestone, without bearing any overall responsibility for the subsequent clinical or market access success of the drug. As such, the different organizational units tend to operate in "silos" [17-19] that develop a "throw-over-the-wall" mentality at the hand-over points. This can be hugely inefficient if seemingly promising products that meet interim safety and efficacy goals lead to commercial failures if the end customer's criteria are not taken into account early on and incorporated into the clinical development plan [20].

The high costs associated with the development process in the pharmaceutical industry can pose a third critical obstacle to collaboration across the different organizational units. Given the high cost of conducting clinical trials, which often run into the hundreds of millions [7], the different business units (e.g., therapeutic areas) within the company often have to compete for very scarce resources. To secure their budget and, therefore, their future development, business units might convey overly optimistic forecasts about the technical and commercial success of a given drug [21]. Because of the highly specialized nature of those forecasts, it is not a trivial exercise for someone outside the business unit to challenge them or the methods used to reach them. Clearly, this is extremely inefficient, as those forecasts are bound to fail in later stages, after having consumed an enormous amount of resources.

Taken together, the three key characteristics of the pharmaceutical industry (high failure rates, long development times, and huge development costs) can create an environment in which there is misalignment in incentives between specific individuals or groups and the overall organization. When that happens, collaboration suffers and development teams focus on the short-term viability (or competence) of their narrowly defined "silo", rather than contributing to the end goal: developing customer-focused medicines.

\section{Behavioral}

Among the most pervasive managerial behaviors is the practice of "throwing good money after bad". As noted in Cooper [22]: "projects get a life of their own and become like express trains, slowing down at the stations, but never with the intention of stopping until they reach their ultimate destination, market launch". Royer [23] also analyses a number of product failures, highlighting that development teams kept going even though there were clear and consistent signs pointing to a near-certain failure. For example, during the development of a novel lens, both the regulatory authorities and opticians expressed clear concerns about its benefits. However, the development team chose to ignore them [23].

The seminal work of Staw [24] demonstrated that subjects were likely to continue making investments in failing projects, despite evidence of negative performance. Importantly, more money was invested in a project when the subjects themselves, rather than an outsider, were also responsible for earlier funding decisions. This self-justification effect has been 
replicated in a number of subsequent experiments $[25,26]$.

A second important bias that prevents efficient decision-making is the confirmatory bias. Here, it is not the past involvement of the decision-maker that biases their future actions. Instead, their current beliefs affect how they seek new information about the project. Specifically, cognitive studies have shown that people tend to seek information that confirms their existing beliefs [27, 28], and at the same time heavily discount information that contradicts those beliefs [29].

As discussed earlier, the R\&D process in the pharmaceutical industry is a complex endeavor that requires highly specialized expertise. Moreover, developing ground-breaking science for unmet medical needs requires an extraordinary amount of perseverance and commitment to succeed. However, those very qualities can often lead to overconfidence [30]: scientists become "true-believers" that their compound will be successful, despite all the evidence against it. This creates an emotional attachment that further amplifies self-justification and confirmatory bias. As a result, the scientists (or the specific development team) develop a "groupthink" mentality [31] where the team isolates itself from the rest of the organization and defends its product by being overly optimistic or by focusing on a narrow set of criteria that might not include the end customer's needs.

\section{Organizational}

Developing and marketing new products is a truly interdisciplinary process that involves a range of individuals from different organizational functions. Research has shown that employees' beliefs and values, and hence their behaviors, are largely driven by the specific functions or groups they belong to. This is expressed in Dougherty [19]: "Departmental thought worlds partition the information and insights. Each has a distinct system of meaning which colors its interpretation of the same information, selectively filters technology-market issues, and produces a qualitatively different understanding of product innovation".

The existence of an insular culture in which people work closely with and learn only from their own group, while excluding those outside the group, is a major barrier to collaboration, and ultimately to product performance. The effect is clearly demonstrated in Hansen et al. [32] who studied 121 product development teams at Hewlett-Packard: the study identified certain teams that only sought solutions within their own team rather than reaching out to other divisions (even when the problem at hand required an interdisciplinary solution). Interestingly, the data also revealed that when teams did reach out to other divisions, they did not necessarily reach out to the ones that would have the highest expertise for their particular problem. People tend to approach those they know and have good relationships with [33] or those they feel most comfortable with [34].

Such organizational barriers to cooperation become even more critical in highly complex development environments such as the pharmaceutical industry. First, the specialized nature of the different processes and tasks creates a direct, and at first sight unavoidable, barrier to collaboration. Specifically, development teams in the pharmaceutical industry might consist of experts from target and drug discovery teams (from synthetic organic chemistry to molecular and cellular biology), clinical teams (from pharmacodynamics and toxicology to pharmacokinetics), and regulatory experts. At the same time, more commercially focused teams consist of experts spanning market access, marketing, medical affairs, finance, and corporate affairs, as well as business development teams that identify the most promising opportunities from the external environment. These individuals are experts in their own area, and possess knowledge and experience that cannot be easily codified or quantified. The organizational literature refers to such tacit knowledge as "sticky": and transferring it across organizational functions is not a trivial task $[35,36]$. Hence, an integrated decision-making process has to rely on the collaboration and effective participation of all teams. 


\section{FRAMEWORK TO OVERCOME THE CHALLENGES AND PROMOTE A CUSTOMER-DRIVEN DEVELOPMENT PROCESS}

\section{Addressing Economic Barriers}

Firstly, a customer-driven mentality requires a focus on the end goal (i.e., commercial success), and as such, a decision-making process that will effectively filter products that will ultimately not meet the customer's requirements. For the pharmaceutical industry, this implies that incentives for the development teams need to be designed in such a way that they promote truth-seeking experimentation, rather than experiments that would accomplish short-term milestones. To overcome such misaligned incentives, recent research has shown that motivating the right kind of experimentation requires a culture with tolerance for failure [37-39]. In practice, and specifically for the pharmaceutical industry, a tolerance for failure would require a stronger emphasis on rewarding the decision-making process itself and not just the interim outcomes. Such process-based metrics encourage employees to focus on the tasks that generate the highest value for the organization, even when those tasks cannot be directly observed and measured [40].

A second key step towards a more customer-centered development process is to facilitate transparency in the resource allocation process. Sharpe and Keelin [21] emphasize the importance of an integrated framework that enables transparency and consistency in the assumptions and assessment criteria that different business units use to assess their $R \& D$ projects. In the example they describe, the key task of the development teams was not to prepare reports that defended their projects, but to demonstrate and document their assumptions and information sources in a succinct and reliable way. On the other hand, the role of senior management was to challenge these valuations in a constructive way, and facilitate a discussion in which the strengths and weakness of each project were identified not in isolation, but with respect to the $R \& D$ portfolio of the entire organization. This iterative and complementary relationship between a top-down (where senior management sets the priorities) and a bottom-up approach (where the development teams provide the critical information) is essential for effective project portfolio selection $[41,42]$.

Lastly, incentive schemes should strike a balance between bonuses based on individual or unit-specific performance and those based on company-wide performance. The latter are particularly effective in enabling better collaboration and knowledge sharing, particularly among competing groups [43, 44]. Huckman et al. [45] describe in detail how Wyeth pharmaceuticals restructured its incentive and reward systems, so that the bonuses of all eligible scientists in the development teams were based on the degree to which the entire organization achieved its objectives. The key objective of this initiative was to motivate the development teams to look beyond their departmental "silos" and strengthen synergies across the various therapeutic areas.

\section{Addressing Behavioral Biases}

The first step in overcoming any behavioral bias is the realization that we are all susceptible to them. This is far from trivial. As noted in Russo and Schoemaker ([30] and references therein), most humans, including managers, have a rather poor understanding of the limits of their knowledge. According to Lovallo and Kahneman [46], such overconfidence often leads managers to believe that they can overcome the challenges in executing a project, even when there is clear evidence to the contrary. This is also consistent with Hammond et al. [47] and Sengupta et al. [48] who argue that even the most experienced and intelligent managers are susceptible to behavioral decision traps. As such, a key recommendation in all of the above studies is that decision-makers should realize the limitations of relying solely on their own judgment, and instead seek to validate it through objective feedback from other units or from outside the organization. It is important that people look at the data without being 
influenced by their role in the project or their personal connections with others working on the project $[23,46,47]$. For the pharmaceutical industry, an advisory board could ensure that challenging questions about the payer's requirements are asked early in the process, rather than a few months before market launch. There are also now more formal options to receive this external customer feedback, with the EMA facilitating a parallel regulatory and Health Technology Assessment (HTA) scientific advice procedure [49].

Secondly, Lovallo and Kahneman [46] stress that companies have to balance optimism and enthusiasm (keeping employees motivated and focused) with realism (ensuring objective forecasts). Along the same lines, Russo and Schoemaker [30] suggest that managers should distinguish between the stages of deciding and doing. The former requires realism and a factbased approach, the latter relies more on motivation and encouragement. For pharmaceutical products, a more objective assessment at the decision stage can be achieved by setting the progression criteria on the basis of lessons learnt from comparable projects that have previously been through the commercialization process. As discussed in Lovallo and Kahneman [46] this "outside view", which relies on data external (rather than internal) to the project, leads to much more objective assessment because "internal view" assessments tend to come with numerous cognitive biases that distort their objectivity. It is important to stress here that external data are not limited to just quantifiable metrics and analytics. Relying only on these is likely to result in companies missing what customers really value [50]. The essence of the external view is that it is inclusive of both qualitative and quantitative insights.

Lastly, senior management should emphasize the need for objective and data-driven estimates about the project's profitability, and particularly about potential factors that might hinder its commercial success. Whereas managers are usually happy to produce optimistic forecasts and reports about their projects, research shows that they are much less effective in seeking information that disconfirms their beliefs [29], especially when this information is not readily available. For pharmaceutical products, it is often difficult to provide precise estimates about a new drug's cost-effectiveness with respect to existing treatments. Models about the economic value of a drug tend to be extremely sensitive to the particular assumptions applied. A potential step forward would be the development of open-source health economic models (for an example, see [51]) that would provide greater transparency and, in turn, more credible and consistent estimations, not only across the different companies but also from the payer's perspective.

\section{Addressing Organizational Barriers}

The first and foremost requirement for a collaborative decision-making process is the existence of unifying goals that require collective effort and commitment from all departments within the organization, including senior management [52]. For this reason, Morgan Stanley decided to introduce peer-evaluation, even in their senior management committees. According to Tom DeLong, senior executive at the time: "Operating Committee members who normally did not share the important knowledge of their divisions realized that at the end of the year they would be evaluating one another. All of a sudden they began to share more information, knowing the consequences at year-end for their evaluations if they didn't" [52]. For the drug commercialization process, this implies that even though the overarching governance body consists of individuals from different functions, it should maintain a clear and strong focus on the end customer's needs [53].

The second important step towards overcoming an insular culture is the development of so-called T-shaped management [54]: managers who can simultaneously excel in their own area (the vertical part of the " $\mathrm{T}$ ") and support collaboration across the different areas (the horizontal part). Such behavior is in sharp contrast with that of "lone star" managers, who only focus on their own performance, or that of "corporate butterflies", who seemingly collaborate with everyone in the organization but without serving a specific purpose. Fostering a T-shaped 
management approach means that collaboration needs to be measured and evaluated. Hansen et al. [55] discuss how management consultancy Bain and Company directly evaluates their partners on how much they have helped other colleagues by collecting detailed data on their collaborative activities. This evaluation comprises a significant part of the partner's annual compensation (up to 25\%). The concept of encouraging T-shaped managers is also consistent with a number of other studies that demonstrate the importance of cross-functional collaboration in new product development settings $[8,56]$. Such a cross-functional management approach is also imperative for managers in the pharmaceutical industry who need to be able to communicate effectively, both with functional experts and across functions.
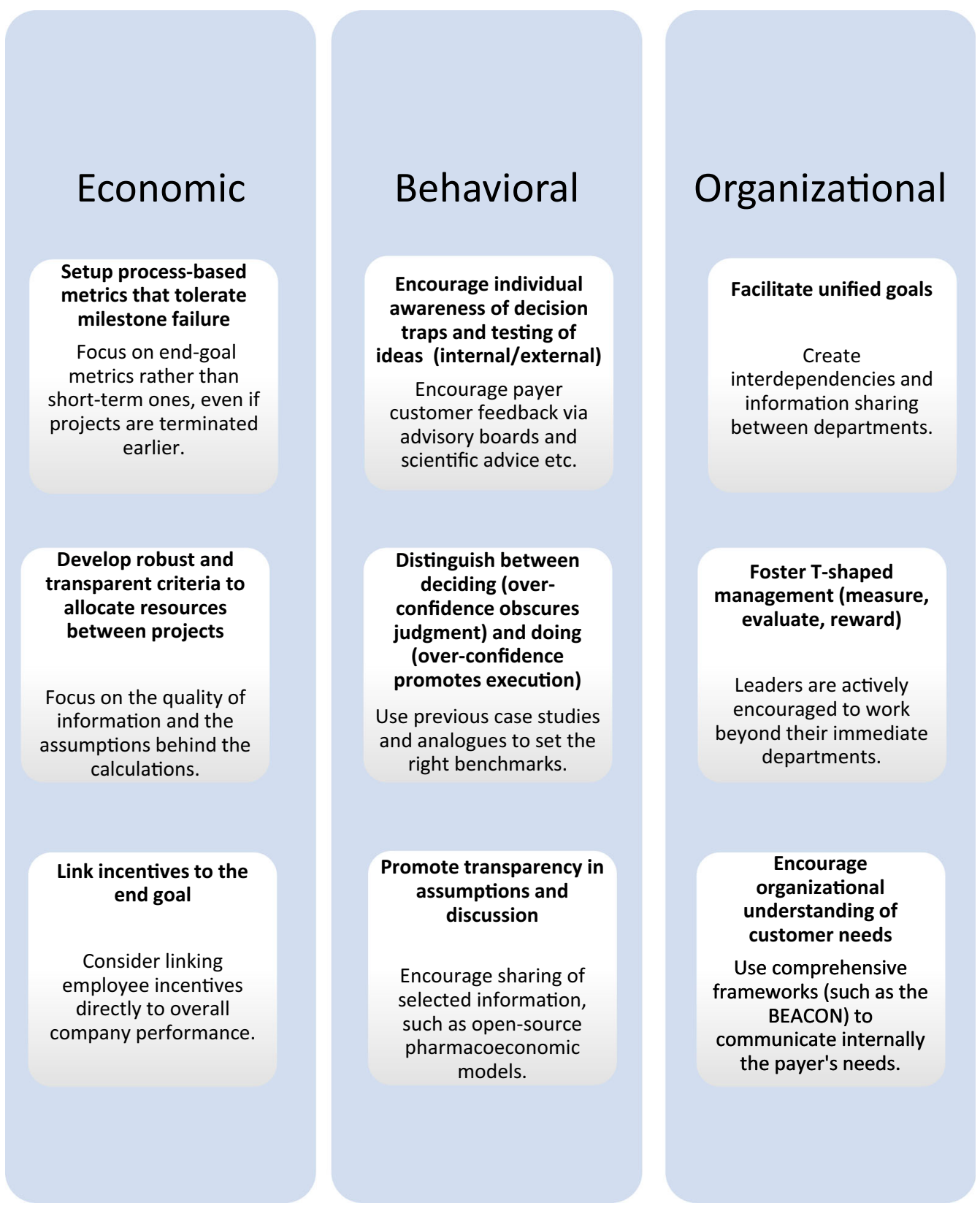

Fig. 1 Actions to address barriers to customer-focused drug development 
Lastly, companies should aim to create bridges between business units that traditionally were perceived as "distant" and cultivate the exchange of knowledge that would allow the creation of a common perspective: how each unit contributes to the customer-driven strategy of the company. For example, Dunlop et al. [53] developed a conceptual framework to address the key needs of the payer and, therefore, overcome potential reimbursement hurdles. Importantly, the framework accounts for both technical and commercial aspects. The mnemonic BEACON (Burden/target population, Environment, Affordability/value, Comparator, Outcomes, Number of studies/quality of evidence) was developed to promote the understanding of the payer's needs throughout the pharmaceutical organization. The diversity in the criteria reflects the different skills required to appropriately assess this and, in turn, the reason why the existence of a cross-functional team is an integral part of a successful commercialization process. Moreover, a key enabler for such integration is that it is embraced and perceived as a fair process [57] by all the teams involved in it. As discussed in Loch and Tapper [40], a fair process is necessary as it helps employees overcome suspicion and accept changes by engaging them (asking for input), explaining the choices (using consistent criteria), and setting clear expectations (what needs to be achieved in the future).

\section{CONCLUSIONS: SUMMARIZING THE FRAMEWORK}

The proposed framework is presented in Fig. 1. It shows the three key categories of barriers to collaboration (economic, behavioral, and organizational) together with specific actions that pharmaceutical companies (and others) can take to address these and enable a more customer-focused development process.

\section{ACKNOWLEDGEMENTS}

This study was funded by Mundipharma International Limited. Editorial services were provided by Dr. Joanna Todd (Stellar Medical Communications Limited) and were funded by Mundipharma International Limited. We would like to thank Antony Mattessich (Managing Director Mundipharma International) for actively supporting and encouraging research towards customer-focused drug development. The article processing charges and Open Access fee associated with this publication were funded by Mundipharma International Limited. All named authors meet the International Committee of Medical Journal Editors (ICMJE) criteria for authorship for this manuscript, take responsibility for the integrity of the work as a whole, and have given final approval for the version to be published.

Disclosures. William Dunlop is an employee of Mundipharma International Limited. Nektarios Oraiopoulos is an employee of the University of Cambridge. Judge Business School Executive Education Ltd received funding for the literature search and analysis.

Compliance with Ethics Guidelines. This article does not contain any new studies with human or animal subjects performed by any of the authors.

Open Access. This article is distributed under the terms of the Creative Commons Attribution-NonCommercial 4.0 International License (http://creativecommons.org/licenses/ by-nc/4.0/), which permits any noncommercial use, distribution, and reproduction in any medium, provided you give appropriate credit to the original author(s) and the source, provide a link to the Creative Commons license, and indicate if changes were made. 


\section{REFERENCES}

1. Johnson A. Insulin flop costs Pfizer \$2.8 billion. 2007. http://www.wsj.com/articles/SB1192690719931632 73. Accessed 17 August 2016.

2. Gutierrez C. Pfizer washes its hands of Exubera. 2007. http://www.forbes.com/2007/10/18/pfizer-earningscloser-markets-equities-cx_cg_1018markets47.html. Accessed 17 August 2016.

3. Rao A, Purkayastha D. "Exubera fiasco: what went wrong?", teaching note 508-115-8. Hyderabad: ICMR Center for Management Research; 2008.

4. National Institute for Health and Care Excellence. Bevacizumab in combination with paclitaxel and carboplatin for first-line treatment of advanced ovarian cancer. 2013. https://www.nice.org.uk/ guidance/ta284?unlid $=5013752442016211165322$. Accessed 17 August 2016.

5. National Institute for Health and Care Excellence. NICE issues final guidance aflibercept for colorectal cancer. 2014. https://www.nice.org.uk/news/pressand-media/nice-issues-final-guidance-aflibercept-forcolorectal-cancer. Accessed 17 August 2016.

6. Harrison RK. Phase II and phase III failures: 2013-2015. Nat Rev Drug Discov. 2016;15(12): 817-8.

7. DiMasi JA, Grabowski HG, Hansen RW. Innovation in the pharmaceutical industry: new estimates of R\&D costs. J Health Econ. 2016;47:20-33.

8. Griffin A, Hauser JR. Patterns of communication among marketing, engineering and manufacturing-a comparison between two new product teams. Manag Sci. 1992;38(3):360-73.

9. Olson EM, Walker OC Jr, Ruekert RW, Bonner JM. Patterns of cooperation during new product development among marketing, operations and R\&D: implications for project performance. J Prod Innov Manag. 2001;18(4):258-71.

10. Slater SF, Mohr JJ, Sengupta S. Radical product innovation capability: literature review, synthesis, and illustrative research propositions. J Prod Innov Manag. 2014;31(3):552-66.

11. Calantone RJ, Schmidt JB, Di Benedetto CA. New product activities and performance: the moderating role of environmental hostility. J Prod Innov Manag. 1997;14(3):179-89.

12. DiMasi JA, Feldman L, Seckler A, Wilson A. Trends in risks associated with new drug development: success rates for investigational drugs. Clin Pharmacol Ther. 2010;87(3):272-7.
13. Munos B. Lessons from 60 years of pharmaceutical innovation. Nat Rev Drug Discov. 2009;8(12): 959-68.

14. Morgan P, Van Der Graaf PH, Arrowsmith J, et al. Can the flow of medicines be improved? Fundamental pharmacokinetic and pharmacological principles toward improving phase II survival. Drug Discov Today. 2012;17(9/10):419-24.

15. Cook D, Brown D, Alexander R, et al. Lessons learned from the fate of AstraZeneca's drug pipeline: a five-dimensional framework. Nat Rev Drug Discov. 2014;13(6):419-31.

16. Pharmaceutical Research and Manufacturers of America (PhRMA). Biopharmaceutical research \& development: the process behind new medicines. 2015. http:// www.phrma.org/report/biopharmaceutical-researchand-development-the-process-behind-new-medicines. Accessed 16 Sept 2016.

17. Hauser JR, Simester DI, Wernerfelt B. Internal customers and internal suppliers. J Mark Res. 1996;33(3):268-80.

18. Souder WE. Achieving organizational consensus with respect to $\mathrm{R} \& \mathrm{D}$ project selection criteria. Manag Sci. 1975;21(6):669-81.

19. Dougherty D. Interpretive barriers to successful product innovation in large firms. Organ Sci. 1992;3(2):179-202.

20. Cooper RG. Third-generation new product processes. J Prod Innov Manag. 1994;11(1):3-14.

21. Sharpe $\mathrm{P}$, Keelin T. How SmithKline Beecham makes better resource-allocation decisions. Harv Bus Rev. 1997;76(2):45-6.

22. Cooper RG. Winning at new products: accelerating the process from idea to launch. Cambridge: Addison-Wesley; 2001.

23. Royer I. Why bad projects are so hard to kill. Harv Bus Rev. 2003;81(2):48-56.

24. Staw BM. Knee-deep in the big muddy: a study of escalating commitment to a chosen course of action. Organ Behav Hum Perform. 1976; $16: 27-44$.

25. Bazerman MH, Beekun RI, Schoorman FD. Performance evaluation in a dynamic context: a laboratory study of the impact of a prior commitment to the rate. J Appl Psychol. 1982;67(6):873-6.

26. Bazerman MH, Giuliano T, Appelman A. Escalation of commitment in individual and group decision making. Organ Behav Hum Perform. 1984;33(2): 141-52. 
27. Jonas E, Schulz-Hardt S, Frey D, Thelen N. Confirmation bias in sequential information search after preliminary decisions: an expansion of dissonance theoretical research on selective exposure to information. J Pers Soc Psychol. 2001;80(4):557-71.

28. Bolton LE. Stickier priors: the effects of nonanalytic versus analytic thinking in new product forecasting. J Mark Res. 2003;40(1):65-79.

29. Biyalogorsky E, Boulding W, Staelin R. Stuck in the past: why managers persist with new product failures. J Mark. 2006;70:108-21.

30. Russo JE, Schoemaker PJH. Managing overconfidence. Sloan Manag Rev. 1992;33(2):7-17.

31. Janis IL. Groupthink: psychological studies of policy decisions and fiascoes. Boston: Houghton Mifflin; 1982.

32. Hansen MT, Mors ML, Løvås B. Knowledge sharing in organizations: multiple networks, multiple phases. Acad Manag J. 2005;48(5):776-93.

33. Hansen MT, Løvås B. How do multinational companies leverage technological competencies? Moving from single to interdependent explanations. Strateg Manag J. 2004;25(8/9):801-22.

34. Casciaro T, Lobo MS. Competent jerks, lovable fools, and the formation of social networks. Harv Bus Rev. 2005;83(6):92-9.

35. Zander U, Kogut B. Knowledge and the speed of the transfer and imitation of organizational capabilities: an empirical test. Organ Sci. 1995;6(1):76-92.

36. Szulanski G. Exploring internal stickiness: impediments to the transfer of best practice within the firm. Strateg Manag J. 1996;17(S2):27-43.

37. Azoulay P, Zivin JSG, Manso G. Incentives and creativity: evidence from the academic life sciences. RAND J Econ. 2011;42(3):527-54.

38. Ederer F, Manso G. Is pay for performance detrimental to innovation? Manag Sci. 2013;59(7):1496-513.

39. Tian X, Wang TY. Tolerance for failure and corporate innovation. Rev Financ Stud. 2014;27(1):211-55.

40. Loch $\mathrm{CH}$, Tapper UAS. Implementing a strategy-driven performance measurement system for an applied research group. J Prod Innov Manag. 2002;19(3):185-98.

41. Terwiesch C, Ulrich K. Managing the opportunity portfolio. Res Technol Manag. 2008;51(5):27-38.

42. Loch C, Kavadias S. Implementing strategy through projects. In: Morris PWG, Pinto J, Söderlund J, editors. The Oxford handbook of project management. Oxford: Oxford University Press; 2011. p. 224-51.

43. Birkinshaw J. Strategies for managing internal competition. Calif Manag Rev. 2001;44(1):21-38.

44. Schlapp J, Oraiopoulos N, Mak V. Resource allocation decisions under imperfect evaluation and organizational dynamics. Manag Sci. 2015;61(9):2139-59.

45. Huckman RS, Pisano GP, Rennella M. Wyeth pharmaceuticals: spurring scientific creativity with metrics. Harvard Business School Case 607-008. 2010.

46. Lovallo D, Kahneman D. Delusions of success: how optimism undermines executives' decisions. Harv Bus Rev. 2003;81(7):56-63.

47. Hammond JS, Keeney RL, Raiffa H. The hidden traps in decision making. Harv Bus Rev. 1998;76(5): 47-58.

48. Sengupta K, Abdel-Hamid TK, Van Wassenhove LN. The experience trap. Harv Bus Rev. 2008;86(2): 94-101.

49. European Medicines Agency. "Best practice guidance for the parallel regulatory-HTA scientific advice procedure". 2016. http://www.ema.europa. eu/docs/en_GB/document_library/Regulatory_ and_procedural_guideline/2016/03/WC500203944. pdf. Accessed 17 August 2016.

50. Christensen C, Hall T, Dillon K, Duncan D. Know your customers' "jobs to be done". Harv Bus Rev. 2016;94(9):54-62.

51. Sullivan W, Hirst M, Beard S, et al. Economic evaluation in chronic pain: a systematic review and de novo flexible economic model. Eur J Health Econ. 2016;17(6):755-70.

52. Hansen MT. Collaboration: how leaders avoid the traps, create unity, and reap big results. Boston: Harvard Business Press; 2009.

53. Dunlop WCN, Mullins CD, Pirk O, et al. BEACON: a summary framework to overcome potential reimbursement hurdles. PharmacoEconomics. 2016. doi:10.1007/s40273-016-0427-7.

54. Hansen MT, von Oetinger B. Introducing T-shaped managers: knowledge management's next generation. Harv Bus Rev. 2001;79(3):106-16.

55. Hansen MT, Nohria N, Tierney T. What's your strategy for managing knowledge? Harv Bus Rev. 1999;77(2):106-16. 
56. Troy LC, Hirunyawipada T, Paswan AK. Cross-functional integration and new product success: an empirical investigation of the findings. J Mark. 2008;72(6):132-46.
57. Kim WC, Mauborgne R. Fair process: managing in the knowledge economy. Harv Bus Rev. 2003;81(1):127-36. 